\title{
Cognitive Characteristics of Attention-Deficit Hyperactivity Disorder in Korean Wechsler Intelligence Scale for Children-Fourth Edition: Focused on General Ability Index and Cognitive Proficiency Index
}

\author{
Min-Je Goo ${ }^{1,2}$, Sang-Woo Oh ${ }^{1,2}$, Sang-Yeol Lee ${ }^{1,2}$, \\ Young-Suk Paik ${ }^{1,2}$, Ji-Hea Lee ${ }^{2,3}$, and Kyu-Sic Hwang ${ }^{1,2}$ \\ ${ }^{1}$ Department of Psychiatry, School of Medicine, Wonkwang University, Iksan, Korea \\ ${ }^{2}$ Wonkwang Brain, Behavior and Mental Health Institute, Iksan, Korea \\ ${ }^{3}$ Wisdom Psychiatric Clinic, Seoul, Korea
}

\section{한국 웩슬러 아동 지능검사-4판(K-WISC-IV)에서 일반능력 지표(GAI)의 임상적 유용성: 주의력결핍 과잉행동장애 아동을 대상으로}

구민제 ${ }^{1,2}$, 오상우 ${ }^{1,2}$, 이상열 ${ }^{1,2}$, 백영석 ${ }^{1,2}$, 이지혜 ${ }^{2,3}$, 황규식 ${ }^{1,2}$

원광대학교 의과대학 정신건강의학교실, ${ }^{1}$ 원광대학교 뇌행동정신건강연구소, ${ }^{2}$ 지혜정신건강의학과 ${ }^{3}$

\begin{abstract}
Objective: The purpose of this study was to investigate the clinical utility of the Korean Wechsler Intelligence Scale for Children-Fourth Edition (K-WISC-IV) and General Ability Index (GAI) in children with attention-deficit hyperactivity disorder (ADHD).

Methods: The GAI and Full Scale IQ (FSIQ) in K-WISC-IV were compared for a group of 79 children with ADHD and 234 normal children. The mean differences within each group were testified by the t-test and Cohen's $\mathrm{d}$. The Wechsler's descriptive classifications for each GAI and FSIQ score were analyzed in the children with ADHD. Additionally, the critical value for the difference between the means of the FSIQ and GAI was calculated in the normal children.

Results: The score on the GAI was higher than that on the FSIQ in the children with ADHD, with a mean difference of 2.19 points. The implications of these findings are discussed regarding the cognitive characteristics of the children with ADHD. According to Wechsler's descriptive classification, the application of the GAI showed a lower (7.59\%), equal (61.65\%) or higher (60.76\%) prevalence of ADHD compared to the application of FSIQ.

Conclusion: The implications of these findings are discussed regarding the cognitive characteristics of the children with ADHD. The GAI reflecting the attention and behavioral problems in children with ADHD could be utilized as an alternative global intelligence estimate that considers their potential for development.
\end{abstract}

Key Words: K-WISC-IV; ADHD; Full Scale IQ; General Ability Index; Cognitive Proficiency Index.

Received: August 19, 2016 / Revision: October 18, 2016 / Accepted: November 25, 2016

Address for correspondence: Sang-Woo Oh, Department of Psychiatry, School of Medicine, Wonkwang University, 895 Muwang-ro, Iksan 54538 , Korea Tel: +82-63-859-1049, Fax: +82-63-857-1043, E-mail: ps15@wonkwang.ac.kr

서 론

2013년에 새롭게 개정된 정신질환의 진단 및 통계 편람 제 5 판(Diagnostic and Statistical Manual of Mental Disorders

This is an Open Access article distributed under the terms of the Creative Commons Attribution Non-Commercial License (http://creativecommons.org/licenses/by-nc/3.0) which permits unrestricted non-commercial use, distribution, and reproduction in any medium, provided the original work is properly cited. fifth edition, DSM-5)에서는 주의력결핍 과잉행동장애(attention-deficit hyperactivity disorder, ADHD)의 진단 기준 을 수정하였다. ${ }^{1 .}$ 수정된 부분 중에서 발병 시기의 연령 기준이 '7세 이전에서 '12세 이전'으로 변경되었으며, 성인 $\mathrm{ADHD}$ 를 고 려하여 따로 증상 역치의 기준이 마련되었다. 이러한 변화는 $\mathrm{ADHD}$ 의 발달과 경과가 아동 및 청소년기에 걸쳐서 다양하 게 발생할 수 있으며, 성인기까지 지속될 수 있음을 시사한다. 
한국에서도 소아청소년의 ADHD 유병률은 7 10\%에 이르며, 실제 병원장면에서 치료를 받는 학생들의 비율은 매년 $4.24 \%$ 씩 증가하고 있다. ${ }^{2,3} \mathrm{DSM}-5$ 에서는 $\mathrm{ADHD}$ 가 기능 또는 발달 을 저해하는 부주의 또는 과잉행동-충동성 증상들이 지속적 으로 나타나는 것을 특징으로 하고 있으며, 이에 따른 결과로 주의력, 수행기능 또는 기억력을 포함하는 검사들에서 인지적 문제가 나타날 수 있음을 밝히고 있다. ${ }^{1)}$

이러한 관점에서 볼 때, $\mathrm{ADHD}$ 아동의 주의력과 관련된 기 능들은 떨어질 것으로 생각된다. ${ }^{4-6)}$ 실제로 주의력을 측정하 는 검사들에서 $\mathrm{ADHD}$ 아동의 수행기능은 저하되어 있었다. 예로, 스트룹 검사(Stroop test), 아동 색 선로 검사(Children's Color Trails Test), 주의력 장애 진단 시스템(Attention Diagnostic System), 연속수행과제(Continuous Performance Test) 등과 같은 검사들을 이용한 선행 연구들은 $\mathrm{ADHD}$ 아동의 선 택적 주의력(selective attention), 지속적 주의력(sustained attention), 정보처리 과정(information processing)에서 결함 이 있으며, 통제기능도 저하가 있을 수 있음을 밝혔다..$^{7-9)}$ 더 불어 대표적인 지능검사로 알려져 있는 웩슬러 아동 지능검 사(Wechsler's Intelligence Scale, WISC)도 ADHD 아동을 대상으로 주의 및 정보처리 기능을 평가해왔다. ${ }^{10)} \mathrm{WISC}$ 는 아 동의 전반적인 지적 능력을 전체검사 점수(Full Scale IQ, FSIQ) 를 통해서 평가할 수 있으며 판(edition)에 따라서 FSIQ를 구성하는 하위 요인들이 조금씩 변하였다. WISC-Revised $(\mathrm{WISC}-\mathrm{R})^{11)}$ 에서는 $\mathrm{ADHD}$ 아동의 산수, 기호쓰기, 숫자 소 검사들의 점수들이 저하되어 있었으며, WISC-III에서는 $\mathrm{ADHD}$ 아동의 주의산만(freedom from distractibility, FD) 요인이 FSIQ보다 유의하게 저하되어 있었다. ${ }^{12,13)}$ 또 다른 연 구들에서도 숫자와 산수 소검사의 점수가 정상 아동 집단과 비교하여 유의하게 저하되어 있었다. ${ }^{14,15)} \mathrm{WISC}-\mathrm{IV}^{16)}$ 는 FSIQ 외에 네 개의 주요 인지기능 지표- 언어이해 지표(Verbal Comprehension Index, VCI), 지각추론 지표(Perceptual Reasoning Index, PRI), 작업기억 지표(Working Memory Index, WMI), 처리속도 지표(Processing Speed Index, PSI)-와 함께 일반능력 지표(General Ability Index, GAI), 인지효능 지표 (Cognitive Proficiency Index, CPI)로 구성되어 있어서 ADHD 증상들에 더 좋은 민감도를 가질 수 있다. ${ }^{17,18)} \mathrm{WISC}-\mathrm{IV}$ 를 이 용한 연구에서는 $\mathrm{ADHD}$ 아동은 $\mathrm{VCI}, \mathrm{PRI}$ 와 비교하여 PSI 와 WMI에서의 수행능력이 유의하게 저하되어 있었다. ${ }^{16-18)}$ 그리하여 VCI와 PRI로 구성되는 GAI와 PSI, WMI로 구성 되는 $\mathrm{CPI}$ 는 $\mathrm{ADHD}$ 아동의 인지적 특성을 이해하는데 도움이 되는 것으로 나타났다. ${ }^{17)}$ 정상 아동 집단과 비교하여 $\mathrm{ADHD}$ 아동의 FSIQ가 저하되어 있을 뿐만 아니라, 집단 내에서의 지 능 프로파일 분석에서도 FSIQ와 GAI가 유의한 차이를 보이
고 있으며, GAI와 CPI도 유의한 차이를 보이고 있었다. ${ }^{17-19)}$

현재 국내에서는 WISC-IV가 표준화되어 한국 웨슬러 아 동 지능검사-4판 [Korean-WISC(K-WISC-IV)] ${ }^{20}$ 이 사용되 고 있는데 K-WISC-IV로 위의 국외 선행 연구 결과들을 지 지 또는 반박하는 연구들은 드문 실정이다. 국내에서는 학교 에서 $\mathrm{ADHD}$ 위험군으로 분류된 아동을 대상으로 하여 $\mathrm{K}-$ WISC-IV 결과를 분석하기는 하였지만, 소검사 및 네 개의 주요 인지기능 지표 수준에서 분석하였다. ${ }^{21)}$ 또한, 그 대상이 비임상 집단이었기 때문에 임상장면에 적용하기에는 무리가 따르는 것으로 생각된다. 따라서 임상장면에 있는 $\mathrm{ADHD}$ 아 동을 대상으로 K-WISC-IV를 이용하여 이들의 FSIQ, GAI, $\mathrm{CPI}$ 를 비교해 보고 인지적 특성을 파악할 필요가 있다. 그리 고 이러한 결과가 국외 선행 연구 결과와 일치하는지 타당성 을 검증해 볼 필요가 있다. 본 연구에서는 임상장면에 있는 $\mathrm{ADHD}$ 아동과 통제 집단 아동을 대상으로 K-WISC-IV에 나 타난 FSIQ, GAI, CPI 양상을 분석하여 이를 국외 연구 결과 들과 비교하고 국내에서의 타당성을 검증해 보고자 한다.

\section{방 법}

\section{대 상}

본 연구는 2012년 1월부터 2016년 4월까지 국내의 지방 소 재 대학교병원 정신건강의학과를 방문한 6세 0개월에서 16세 11 개월에 해당하는 아동을 대상으로 하였다. 정신건강의학과 전문의가 아동 및 아동의 부모를 대상으로 임상적 면담과 평 가를 실시하였으며, DSM-IV 및 DSM-5 ADHD 진단 기준에 근거하여 $\mathrm{ADHD}$ 진단을 내렸다. $\mathrm{ADHD}$ 가 아닌 공존하는 다 른 문제들이 인지기능에 미치는 영향을 배제하기 위하여, 지 적 결손이 있는 아동(K-WISC-IV의 FSIQ가 80점 미만), 다른 정신과적 진단(특정학습장애, 주요우울장애, 틱장애, 품행장 애 등)을 받은 아동, 뇌질환의 병력이 있는 아동을 제외하였 다. 최종적으로 23 명의 아동이 선정되었으며 이를 $\mathrm{ADHD}$ 집 단으로 명명하였다. 통제 집단의 경우 선정된 $\mathrm{ADHD}$ 집단과 비교하여 개별적으로 성별, 나이, 지역 변인들이 일치되는 아 동을 $\mathrm{K}-\mathrm{WISC}-\mathrm{IV}$ 표준화 표본에서 무선 추출하였다. 무선 추출 방식은 Predictive Analytics SoftWare(PASW) 18.0.0 (SPSS Inc., Chicago, IL, USA) 프로그램의 케이스 선택 방식 에서 '무작위 표본추출'을 이용하였다. 마찬가지로 최종적으로 23명의 아동이 선정되었으며 이를 대응 통제 집단(matched control group)으로 명명하였다. 즉, 임상 집단과 정상 통제 집단으로 나누어 두 집단을 비교하였으며, 임상 집단 내 분석 도 포함되었다. 본 연구는 소속 대학교병원 기관생명윤리위원 회(Institutional Review Board No. WKUH 201607-HRE- 
072) 심사를 받고 승인을 받은 후에 진행되었다.

\section{도 구}

한국 웩슬러 아동 지능검사-4판(Korean-Wechsler Intelligence Scale for Children-forth edition, K-WISC-IV)

원판인 미국의 WISC-IV ${ }^{16}$ 를 한국판으로 표준화한 한국 웩슬러 아동 지능검사-4판(K-WISC-IV)을 이용하였다. KWISC-IV는 6세 0개월부터 16세 11개월까지의 아동과 청소 년의 인지능력을 평가하기 위해 개별적으로 실시하는 지능검 사이다. 한국 웩슬러 아동 지능검사-3판(K-WISC-III)과 비 교하여 유동적 추론 및 작업기억, 처리속도에 대한 측정을 개 선하기 위하여 새로운 소검사들을 도입하였다. K-WISC-IV 는 아동이 이해하기 쉽도록 지시문이 수정되고 가르치는 문항, 예시 및 연습 문항이 포함되었다. 또한, 더 쉽고 더 어려운 문 항들이 추가되어 극단적인 범위의 인지능력에 있는 아동의 수 행을 보다 정확하게 측정하는 것이 가능하다. 더불어 본 연구 에서 다루어지는 GAI 및 $\mathrm{CPI}$ 를 산출할 수 있으며, 몇 개의 소 검사들로 구성된 임상적 군집에 대한 해석도 가능하다. ${ }^{22)}$ 한 국판 표준화 과정에서 전체 표준화 집단에 대한 FSIQ 및 네 가지 지표에 대한 전체 평균 내적 일치도 계수들은 $0.81(\mathrm{PSI})$ 부터 0.94(FSIQ)까지로 적절하였으며, 검사-재검사 안정수 계 수들도 $0.82(\mathrm{PRI})$ 부터 $0.92\left(\mathrm{FSIQ}\right.$ )까지로 적절하였다. ${ }^{20)}$ 타당 도 검증을 위한 요인 분석에서는 4 요인 모형이 가장 적합한 것으로 나타나 이론에 따른 구성 타당도가 검증되었다.

\section{자료분석}

먼저 집단에 따라서 인구통계학적 정보들에 대한 기술 통 계로 평균 및 표준편차를 산출하였다. 다음으로 $\mathrm{ADHD}$ 집단 과 대응 통제 집단에 따라서 K-WISC-IV에서 나타난 각 지표 점수, 소검사 환산점수들에 대한 평균 및 표준편차를 산출하 였다. $\mathrm{ADHD}$ 집단과 대응 통제 집단을 비교할 때는, $\mathrm{FSIQ}$ 끼 리의 평균 차이, GAI끼리의 평균 차이, CPI끼리의 평균 차이 를 계산하였다. 평균 차이 검증을 위해서는 두 독립표본 t-test 를 실시하였으며, 집단 간의 평균 차이가 유의할 때는 효과 크 기를 Cohen's d를 이용하여 알아보았다. ${ }^{23)}$ 마지막으로 ADHD 아동 집단 내에서 $\mathrm{FSIQ}$ 와 $\mathrm{GAI}$ 의 차이, $\mathrm{FSIQ}$ 와 $\mathrm{CPI}$ 의 차이, $\mathrm{GAI}$ 와 CPI의 차이 값들을 산출하였다. 이 때, 평균 차이 검 증을 위해서 대응표본 t-test를 실시하였으며, 평균 차이가 유 의할 때는 효과 크기를 Cohen's d를 이용하여 알아보았다. ${ }^{23)}$ Cohen's d에서 d의 크기가 0.5 미만일 경우에는 '작은 효과', 0.5 0.8 사이일 때는 '중간 효과', 0.8 이상일 경우에는 '큰 효 과로 알려져 있다.

\section{결 과}

\section{인구통계학적 특성(Table 1)}

$\mathrm{ADHD}$ 집단과 대응 통제 집단의 연구대상자 수는 각각 23 명으로 동일하였다. ADHD 집단의 성별, 나이, 지역에 대응되 는 통제 집단으로 구성하였기에 이 두 집단의 성별 비율은 동 일하였다. 각 집단에서 남아는 17 명으로 $73.9 \%$ 였으며, 여아는 6 명으로 $26.1 \%$ 를 차지하였다. ADHD 집단의 평균 연령은 8.68 세였으며, 대응 통제 집단의 평균 연령은 8.43세였다. $\mathrm{ADHD}$ 아동 집단의 평균 교육 수준은 3.08년이었으며, 대응 통제 집 단의 평균 교육 수준은 3.04년이었다. 연령과 교육 수준에서 두 집단 간 유의한 차이는 없었다.

\section{$\mathrm{ADHD}$ 집단과 대응 통제 집단에 따른 K-WISC-IV의 각 지표 점수, 소검사 환산점수들에 대한 평균 및 표준편차(Table 2)}

먼저 $\mathrm{ADHD}$ 집단의 결과를 살펴보면 다음과 같다. 각 지 표 수준에서(평균 100, 표준편차 15), FSIQ, GAI, CPI는 모두 '평균' 수준으로 나타났으나 GAI가 96.61점으로 가장 높았으 며, 다음으로 FSIQ가 92.00점, 마지막으로 CPI가 91.22점으 로 가장 낮았다. 네 가지 주요 인지기능 지표에 따라 나누어 서 살펴보면(평균 100 , 표준편차 15), PSI를 제외한 VCI, PRI, WMI가 '평균' 수준에 해당하였다. VCI가 98.57점으로 가장 높았으며, 다음으로 PRI가 96.17점, 다음으로 WMI가 94.65 점, 마지막으로 PSI가 86.57점으로 가장 낮았다. 소검사 수준 에서 살펴보면(평균 10 , 표준편차 3), 어휘 소검사(vocabulary) 의 환산점수가 11.35 점으로 가장 높았으며 기호쓰기 소검사 (coding, CD)의 환산점수가 7.35점으로 가장 낮았다.

다음으로 대응 통제 집단의 결과를 살펴보면 다음과 같다. 각 지표 수준에서(평균 100 , 표준편차 15$), \mathrm{FSIQ}, \mathrm{GAI}, \mathrm{CPI}$ 는 모두 '평균' 수준으로 나타났으나 CPI가 103.91점으로 가장 높 았으며, 다음으로 FSIQ가 102.43점, 마지막으로 GAI가 100.74 점이었다. 네 가지 주요 인지기능 지표에 따라 나누어서 살펴 보면(평균 100, 표준편차 15), VCI, PRI, WMI, PSI 모두 '평균' 수준에 해당하였다. PSI가 103.87점으로 가장 높았으며, 다음 으로 WMI가 102.57점, 다음으로 PRI가 102.04점, 마지막으

Table 1. Demographic characteristics of participants by group

\begin{tabular}{lll}
\hline \multicolumn{1}{c}{ Variable } & \multicolumn{1}{c}{$\begin{array}{c}\text { ADHD group } \\
(\mathrm{n}=23)\end{array}$} & $\begin{array}{c}\text { Matched control } \\
\text { group }(\mathrm{n}=23)\end{array}$ \\
\hline Gender, $\mathrm{n}(\%)$ & Male=17 (73.9) & Male=17 (73.9) \\
& Female=6(26.1) & Female=6 (26.1) \\
Age:M (SD), yrs & $8.68(1.80)$ & $8.43(1.85)$ \\
Education:M (SD), yrs & $3.08(2.29)$ & $3.04(1.85)$
\end{tabular}

ADHD: attention-deficit hyperactivity disorder, $M$ : mean, SD: standard deviation, yrs: years 
로 $\mathrm{VCI}$ 가 99.30점으로 가장 낮았다. 소검사 수준에서 살펴보 면(평균 10 , 표준편차 3$), \mathrm{CD}$ (기호쓰기 소검사)의 환산점수가 11.08 점으로 가장 높았으며 이해 소검사(comprehension)의

Table 2. Mean and standard deviation of each index and subtest of K-WISC-IV by group

\begin{tabular}{|c|c|c|}
\hline \multirow{2}{*}{$\begin{array}{l}\text { Subtest or } \\
\text { index }\end{array}$} & $\begin{array}{c}\text { ADHD group } \\
\quad(n=23)\end{array}$ & $\begin{array}{c}\text { Matched control } \\
\text { group }(n=23)\end{array}$ \\
\hline & $M(S D)$ & $M(S D)$ \\
\hline$F S I Q$ & $92.00(8.64)$ & $102.43(12.81)$ \\
\hline GAl & $96.61(12.57)$ & $100.74(11.91)$ \\
\hline CPI & $91.22(9.43)$ & $103.91(14.16)$ \\
\hline $\mathrm{VCl}$ & 98.57 (12.89) & $99.30(11.91)$ \\
\hline PRI & $96.17(12.25)$ & $102.04(12.09)$ \\
\hline WMI & $94.65(12.68)$ & $102.57(15.25)$ \\
\hline PSI & 86.57 (13.72) & $103.87(11.88)$ \\
\hline $\mathrm{BD}$ & $9.87(2.55)$ & $10.00(2.05)$ \\
\hline SI & $9.17(3.10)$ & $10.04(2.44)$ \\
\hline DS & $8.83(2.61)$ & $9.91(3.18)$ \\
\hline PCn & $8.52(2.73)$ & $10.65(2.44)$ \\
\hline$C D$ & 7.35 (3.20) & $11.08(3.09)$ \\
\hline VC & 11.35 (2.95) & $9.87(2.47)$ \\
\hline LN & $9.26(2.77)$ & $10.91(3.03)$ \\
\hline MR & $9.52(2.68)$ & $10.08(3.00)$ \\
\hline $\mathrm{CO}$ & $8.52(2.10)$ & $9.70(2.88)$ \\
\hline SS & $8.04(2.27)$ & $10.22(1.81)$ \\
\hline
\end{tabular}

K-WISC-IV: Korean-Wechsler Intelligence Scale of Childrenfourth edition, $M$ : mean, SD: standard deviation, ADHD: attention-deficit hyperactivity disorder, BD: block design, SI: similarities, DS: digit span, PCn: picture concepts, CD: coding, VC: vocabulary, LN: letter-number sequencing, MR: matrix reasoning, CO: comprehension, SS: symbol search, VCl: Verbal Comprehension Index, PRI: Perceptual Reasoning Index, WMI: Working Memory Index, PSI: Processing Speed Index, FSIQ: Full Scale IQ, GAI: General Ability Index, CPI: Cognitive Proficiency Index
환산점수가 9.70점으로 가장 낮았다.

\section{$\mathrm{ADHD}$ 집단과 대응 통제 집단 간 $\mathrm{FSIQ}, \mathrm{GAI}, \mathrm{CPI}$ 에 대한 평균 차이 검증 결과(Table 3)}

두 집단 간 FSIQ, GAI, CPI 평균 차이를 살펴보았다. FSIQ 의 경우, 대응 통제 집단이 102.43점, $\mathrm{ADHD}$ 집단이 92.00점 으로 대응 통제 집단이 $\mathrm{ADHD}$ 집단보다 10.43점 더 높았다. 그 차이는 통계적으로 유의하였으며 효과 크기는 '큰 효과’였 다 $[\mathrm{t}(44)=-3.24, \mathrm{p}<0.01, \mathrm{~d}=3.24]$. GAI의 경우, 대응 통제 집단 이 100.74점, $\mathrm{ADHD}$ 집단이 96.61점으로 대응 통제 집단이 $\mathrm{ADHD}$ 집단보다 4.13점 더 높았다. 그 차이는 통계적으로 유 의하지 않았다 $[\mathrm{t}(44)=-1.14, \mathrm{p}=0.130] . \mathrm{CPI}$ 의 경우, 대응 통제 집단이 103.91점, $\mathrm{ADHD}$ 집단이 91.22점으로 대응 통제 집단 이 $\mathrm{ADHD}$ 집단보다 12.69점 더 높았다. 그 차이는 통계적으 로 유의하였으며 효과 크기는 '큰 효과'였다 $[\mathrm{t}(44)=-3.58, \mathrm{p}$ $<0.001, \mathrm{~d}=3.57$ ]. 종합해 볼 때, ADHD 집단과 대응 통제 집 단 사이의 FSIQ, GAI, CPI 비교에서 FSIQ와 CPI는 집단 간 에 서로 유의한 차이가 있는 것으로 나타났으며, 그 차이는 모두 '큰 효과’였다.

\section{$\mathrm{ADHD}$ 집단 내에서 FSIQ-GAI, FSIQ-CPI, GAI-CPI, $\mathrm{FSIQ}-\mathrm{CP}$ 의 평균 차이 검증 결과(Table 4)}

마지막으로 $\mathrm{ADHD}$ 집단 내에서 $\mathrm{FSIQ}$ 와 $\mathrm{GAI}$ 의 평균 차이, $\mathrm{GAI}$ 와 $\mathrm{CPI}$ 의 평균 차이, $\mathrm{FSIQ}$ 와 $\mathrm{CPI}$ 의 평균 차이를 살펴보 았다. 먼저 FSIQ와 GAI의 평균 차이는 -4.61점으로 $\mathrm{GAI}$ 의 평균 점수가 $\mathrm{FSIQ}$ 의 평균 점수보다 높았다. 그 차이는 통계적 으로 유의하였으며, 효과 크기는 '큰 효과’로 나타났다 $[\mathrm{t}(21)=$

Table 3. Mean difference for FSIQ, GAl, and CPI of K-WISC-IV between two groups

\begin{tabular}{|c|c|c|c|c|c|c|}
\hline \multirow[t]{2}{*}{ Index } & $\begin{array}{l}\text { ADHD group } \\
\quad(n=23)\end{array}$ & $\begin{array}{l}\text { Matched control } \\
\text { group }(n=23)\end{array}$ & \multirow{2}{*}{$\begin{array}{c}\text { Mean difference } \\
\text { value }^{a}\end{array}$} & \multirow[t]{2}{*}{ t value ${ }^{b}$} & \multirow[t]{2}{*}{$\mathrm{p}$ value } & \multirow[t]{2}{*}{ d value } \\
\hline & $M(S D)$ & $M(S D)$ & & & & \\
\hline FSIQ & $92.00(8.64)$ & $102.43(12.81)$ & -10.43 & -3.24 & $0.001^{* *}$ & 3.24 \\
\hline GAl & $96.61(12.57)$ & $100.74(11.91)$ & -4.13 & -1.14 & 0.130 & - \\
\hline CPI & 91.22 (9.43) & $103.91(14.16)$ & -12.69 & -3.58 & $0.000^{* * *}$ & 3.57 \\
\hline
\end{tabular}

athe difference value is calculated by the mean value of ADHD group minus the one of matched control group, ${ }^{b}$ it is followed by

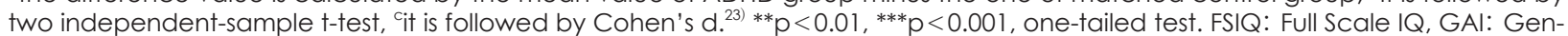
eral Ability Index, CPI: Cognitive Proficiency Index, K-WISC-IV: Korean-Wechsler Intelligence Scale of Children-fourth edition, ADHD: attention-deficit hyperactivity disorder, M: mean, SD: standard deviation

Table 4. Mean difference for FSIQ-GAI, FSIQ-CPI, and GAI-CPI of K-WISC-IV within ADHD group ( $n=23$ )

\begin{tabular}{cccccc}
\hline Index & Mean & Mean difference value & t value $^{a}$ & \multicolumn{2}{c}{$\mathrm{p} \mathrm{value}^{\mathrm{d} \text { value }}$} \\
\hline FSIQ-GAI & $92.00-96.61$ & -4.61 & -3.08 & $0.003^{* *}$ & 4.78 \\
FSIQ-CPI & $92.00-91.22$ & 0.78 & 0.45 & 0.330 & - \\
GAI-CPI & $96.61-91.22$ & 5.39 & 1.77 & 0.05 & 1.77 \\
\hline
\end{tabular}

it is followed by paired t-test, ${ }^{\text {it }}$ is followed by Cohen's $d^{23)}{ }^{* *} \mathrm{p}<0.01$, one-tailed test. FSIQ: Full Scale IQ, GAI: General Ability Index, CPI: Cognitive Proficiency Index, K-WISC-IV: Korean-Wechsler Intelligence Scale of Children-fourth edition, ADHD: attentiondeficit hyperactivity disorder 
$-3.08, \mathrm{p}<0.01, \mathrm{~d}=4.78]$. 다음으로 FSIQ와 CPI의 평균 차이는 0.78 점으로, 그 차이는 통계적으로 유의하지 않았다 $[\mathrm{t}(21)=0.45$, $\mathrm{p}=0.330]$. 마지막으로 GAI와 CPI의 평균 차이는 5.39점으로 $\mathrm{GAI}$ 의 평균 점수가 $\mathrm{CPI}$ 의 평균 점수보다 높았다. 그 차이는 통계적으로 유의하였으며, 효과 크기는 '큰 효과’로 나타났다 $[\mathrm{t}(21)=1.77, \mathrm{p}<0.05, \mathrm{~d}=1.77]$. 종합해 볼 때, $\mathrm{ADHD}$ 집단 내에 서 $\mathrm{GAI}$ 의 평균 점수는 $\mathrm{FSIQ}$ 와 $\mathrm{CPI}$ 의 평균 점수보다 각각 크 고 그 차이는 모두 유의하였다. $\mathrm{FSIQ}$ 와 $\mathrm{CPI}$ 의 평균 점수 차 이는 서로 유의하지 않았다.

\section{고 찰}

본 연구는 $\mathrm{ADHD}$ 아동에서 나타나는 인지적 특성을 $\mathrm{K}-$ $\mathrm{WISC}-\mathrm{IV}$ 의 GAI와 $\mathrm{CPI}$ 를 중심으로 살펴보았다. 연구 결과 에 대한 함의는 아래와 같다.

먼저, K-WISC-IV를 통해 ADHD 아동의 FSIQ, GAI, CPI 양상을 파악할 수 있었다. ADHD 아동의 FSIQ, GAI, CPI는 모두 '평균' 수준으로 나타났으나, 그 크기는 GAI, FSIQ, CPI 순서로 $\mathrm{GAI}$ 점수가 가장 높았으며 $\mathrm{CPI}$ 점수가 가장 낮았다. 이러한 결과는 WISC-IV로 $\mathrm{ADHD}$ 아동의 GAI, FSIQ, CPI 양상을 살펴본 선행 연구 결과들과 일치하는 것이다. ${ }^{17-19)}$ 이러 한 양상을 보이는 이유는 GAI, FSIQ, CPI를 구성하는 주요 인지기능 지표와 각 소검사가 다르기 때문이다. GAI의 경우 $\mathrm{VCI}$ 와 PRI로 구성되는데 $\mathrm{FSIQ}$ 와는 상대적으로 $\mathrm{WMI}$ 와 PSI 의 영향을 적게 받는다. 소검사 수준에서 설명하면, WMI와 PSI를 구성하고 있는 숫자(digit span, DS), CD, 순차연결(letter-number sequencing, LN), 동형찾기(symbol search, SS) 소검사들의 영향을 덜 받기 때문이며, $\mathrm{ADHD}$ 아동은 이러한 소검사들에서 수행 능력이 저하되어 있음을 시사한다. ${ }^{24,25)}$ $\mathrm{ADHD}$ 아동을 대상으로 한 WISC-R 및 WISC-III의 선행 연구들에서도 $\mathrm{DS}$ 와 $\mathrm{CD}$ 의 수행이 저조하여 $\mathrm{FD}$ 지표가 저하 될 수 있음을 설명하였다. ${ }^{13-15)}$ 따라서 $\mathrm{ADHD}$ 아동은 DS, $\mathrm{CD}, \mathrm{LN}, \mathrm{SS}$ 를 포함하고 있는 $\mathrm{CPI}$ 점수가 이들을 포함하지 않는 $\mathrm{GAI}$ 점수보다 적게 나오게 된다.

둘째, K-WISC-IV에서 나타난 ADHD 아동의 FSIQ, GAI, $\mathrm{CPI}$ 특성을 정상 아동 집단과 비교할 수 있었다. 특히, 이번에 설계된 대조 집단에 해당하는 정상 아동들은 $\mathrm{K}-\mathrm{WISC}-\mathrm{IV}$ 표준화 표본 아동 중에서 $\mathrm{ADHD}$ 아동의 성별, 연령, 지역에 대응되며, 무선으로 추출된 것이다. FSIQ, GAI, CPI 비교 결 과를 살펴보면, $\mathrm{ADHD}$ 아동의 FSIQ, GAI, $\mathrm{CPI}$ 는 정상 아동 의 FSIQ, GAI, CPI보다 모두 저하되어 있었다. 이러한 결과는 선행 연구 결과들에서도 나타났으며, $\mathrm{ADHD}$ 아동의 전반적인 지적 능력, 일반능력 및 인지적 효율성이 정상 아동에 비해
저하되어 있음을 시사한다. ${ }^{24,25)}$ 하지만, 이러한 저하가 통계적 으로 유의한 것인지에 대해서 살펴보면, $\mathrm{FSIQ}$ 의 경우 통계적 으로 유의한 차이가 있었다. $\mathrm{ADHD}$ 아동의 전반적인 지적 능 력이 정상 아동의 전반적인 지적 능력보다 유의하게 저하되어 있었으며 그 차이에 대한 효과 크기는 '큰 효과'였다. CPI에서 도 통계적으로 유의한 차이가 나타났다. $\mathrm{ADHD}$ 아동의 인지 적 효율성이 정상 아동의 인지적 효율성보다 유의하게 저하되 어 있었으며 그 차이에 대한 효과 크기는 '큰 효과'였다. 하지 만, $\mathrm{GAI}$ 의 경우 $\mathrm{ADHD}$ 아동의 일반능력이 정상 아동의 일반 능력보다 저하되어 있기는 하였으나 통계적으로 유의하지 않 았다. 이러한 결과들은 $\mathrm{ADHD}$ 아동의 인지적 능력이 언어 및 추론 능력보다는 주의력 및 정보처리 기능에서 결함이 있음을 말해 주고 있으며, K-WISC-IV의 FSIQ, GAI, CPI 양상을 통 해서 잘 확인될 수 있었다.

셋째, $\mathrm{ADHD}$ 아동 집단 내에서 나타나는 $\mathrm{FSIQ}$ 와 $\mathrm{GAI}$ 의 차이, $\mathrm{GAI}$ 와 $\mathrm{CPI}$ 의 차이도 유의한 것으로 나타났으며 이 또 한 이들의 인지적 특성을 반영하고 있었다. 선행 연구에서 살 펴보면 $\mathrm{ADHD}$ 아동의 경우 $\mathrm{GAI}$ 가 $\mathrm{CPI}$ 보다 점수가 높으며, 그 차이는 유의한 것으로 나타났는데 본 연구에서도 그와 일 치하는 양상이 나타났다. 17-19) 그 차이에 대한 효과 크기도 '큰 효과로 나타났다. 뿐만 아니라 FSIQ와 GAI의 평균 차이 검 증에서도 GAI가 $\mathrm{FSIQ}$ 보다 점수가 높고 그 차이는 유의한 것 으로 나타났다. 그 차이에 대한 효과 크기도 '큰 효과’로 나타 났다. 다시 말해서 $\mathrm{ADHD}$ 아동에서 보이는 GAI와 CPI의 차 이, $\mathrm{FSIQ}$ 와 GAI의 차이는 흔히 볼 수 있는 차이가 아니며, $\mathrm{ADHD}$ 아동을 대상으로 한 K-WISC-IV에서 나타나는 인지 적 특성으로 볼 수 있다. 한편, 대응 통제 집단을 대상으로 이 차이들을 검증하였는데, 그 차이들은 통계적으로 유의하지 않았다.

본 연구에서 $\mathrm{ADHD}$ 아동의 인지적 특성을 K-WISC-IV의 $\mathrm{GAI}, \mathrm{CPI}$ 를 통해서 확인할 수 있었지만 몇 가지 제한점과 부 족한 점들이 남아 있다. 먼저, 본 연구가 국외 WISC-IV 선행 연구 결과들과 일치되는 결과들을 보여주었지만, K-WISC$\mathrm{IV}$ 를 이용하여 재검증하는 반복 연구들이 필요할 것으로 생 각된다. 국외 WISC-IV 연구에서도 GAI와 CPI의 차이가 과연 $\mathrm{ADHD}$ 를 진단할 수 있는 정확성에 대한 논쟁은 있었다. ${ }^{18)}$ 국 내에서도 반복 검증을 통하여 타당성을 재검증할 필요가 있 다. 둘째, 통계적으로 유의한 차이가 있는가에 대해서 살펴보 기는 하였지만, 기저율에 대한 조사도 뒤따라야 할 것으로 생 각된다. 즉, $\mathrm{GAI}$ 와 $\mathrm{CPI}$ 의 차이 또는 $\mathrm{FSIQ}$ 와 $\mathrm{GAI}$ 의 차이가 유의하기는 하지만 이러한 차이가 실제 $\mathrm{ADHD}$ 아동 집단에 서 차지하는 누적 백분율을 살펴볼 수 있다면 매우 유용할 것으로 생각된다. ${ }^{26)}$ 본 연구에서 이러한 누적 백분율 조사를 
시도하였으나, 기저율을 반영하기에는 참가자 수가 충분하지 못했기 때문에 제외시켰다. 끝으로, 본 연구에서는 $\mathrm{ADHD}$ 아 동을 대상으로 $\mathrm{GAI}$ 와 $\mathrm{CPI}$ 에 나타난 인지적 특성을 살펴보았 지만, 임상 장면에 있는 다른 아동 집단을 대상으로 활용할 수도 있을 것이다. 이미 국외 연구들에서는 WISC-IV의 GAI 및 $\mathrm{CPI}$ 를 활용하여 영재 아동, 지적장애/학습장애/자폐 스펙 트럼 장애 등이 있는 아동에서 나타나는 GAI와 CPI 특성을 연구한 바 있다. ${ }^{2425)}$ 영재 아동의 경우 GAI보다 FSIQ가 높게 나타나는 경우가 많았으며, 학습장애가 있는 아동의 경우 $\mathrm{ADHD}$ 아동과 마찬가지로 $\mathrm{GAI}$ 가 FSIQ보다 높게 관찰되었 다. 고기능 자폐 아동의 경우 GAI가 FSIQ보다 높았으며, 경도 의 지적장애 아동들은 $\mathrm{GAI}$ 점수가 $\mathrm{CPI}$ 점수에 비해 유의하 게 낮았다.

\section{결 론}

$\mathrm{K}$-WISC-IV에 나타난 ADHD 아동의 인지적 특성을 GAI 와 $\mathrm{CPI}$ 를 중심으로 살펴보았다. 국외 WISC-IV 선행 연구 결 과들과 마찬가지로 $\mathrm{K}-\mathrm{WISC}$ 에서 $\mathrm{ADHD}$ 아동의 $\mathrm{GAI}$ 는 정 상 아동 집단과 비교하여 유의한 차이가 없었으나, FSIQ와 $\mathrm{CPI}$ 는 정상 아동 집단보다 유의하게 낮았다. $\mathrm{ADHD}$ 아동 집 단 내에서 살펴보면 GAI가 가장 높았으며 다음으로 FSIQ, $\mathrm{CPI}$ 가 가장 낮았다. $\mathrm{FSIQ}$ 와 $\mathrm{GAI}$ 의 차이, $\mathrm{GAI}$ 와 $\mathrm{CPI}$ 의 차이 는 유의한 것으로 나타났으며, 정상 아동 집단과 달리 이러한 $\mathrm{GAI}$ 와 $\mathrm{CPI}$ 에서 나타나는 양상으로 $\mathrm{ADHD}$ 아동의 인지적 특 성을 확인할 수 있었다. 따라서 $\mathrm{K}-\mathrm{WISC}-\mathrm{IV}$ 의 GAI 및 $\mathrm{CPI}$ 양상을 통하여 $\mathrm{ADHD}$ 아동이 가지고 있는 인지적 결함을 잘 이해할 수 있으며 인지 훈련 및 학습 계획을 세우는 데도 도 움이 될 것으로 생각된다.

중심 단어: 한국 웩슬러 아동 지능검사-4판;

주의력결핍 과잉행동장애; 전체 $\mathrm{IQ}$; 일반능력 지표;

인지효능 지표.

\section{Conflicts of Interest}

The authors have no financial conflicts of interest.

\section{REFERENCES}

1) American Psychiatric Association. Diagnostic and statistical manual of mental disorders: DSM-5. 5th ed. Washington, DC: American Psychiatric Association;2013. p.103-106.

2) Lee DH. Estimated prevalence of ADHD symptoms and relationships of ADHD symptoms with depression, anxiety, stress, and school maladjustment. Korea J Couns 2009;10:2397-2419.

3) National Health Insurance Service. Presentation of analysis of health insurance, 2013 [cited 2016 Aug 10]. Available from: http:// www.nhis.or.kr/bbs7/boards/B0039/13958.
4) Barkley RA. Psychological assessment of children with ADHD. In: Barkley RA, editor. Attention-deficit hyperactivity disorder: a handbook for diagnosis and treatment. 4th ed. New York: Guilford Press; 2014. p.455-474.

5) Kaufman AS, Lichtenberger EO. Assessing adolescent and adult intelligence. 3rd ed. New Jersey: John Wiley \& Sons;2005. p.225-236.

6) Flanagan DP, Harrison PL. Contemporary intellectual assessment: theories, tests, and issues. 3rd ed. New York: Guilford Press;2012. p.687-707.

7) Huang-Pollock CL, Karalunas SL, Tam H, Moore AN. Evaluating vigilance deficits in ADHD: a meta-analysis of CPT performance. J Abnorm Psychol 2012;121:360-371.

8) Koo HJ, Shin MS. A standardization study of Children's Color Trails Test (CCTT). J Korean Acad Child Adolesc Psychiatry 2008;19:2837.

9) Schwartz K, Verhaeghen P. ADHD and Stroop interference from age 9 to age 41 years: a meta-analysis of developmental effects. Psychol Med 2008;38:1607-1616.

10) Mayes SD, Calhoun SL. WISC-IV and WISC-III profiles in children with ADHD. J Atten Disord 2006;9:486-493.

11) Wechsler D. Wechsler Intelligence Scale for Children-Revised. New York: Psychological Corporation;1974.

12) Wechsler D. Wechsler Intelligence Scale for Children (WISC-III): Manual. San Antonio, TX: Psychological Corporation;1991.

13) Schwean VL, Saklofske DH. WISC-III assessment of children with attention deficit/hyperactivity disorder. In: Prifitera A, Saklofske DH, editors. WISC-III clinical use and interpretation: scientistpracticioner perspectives. San Diego: Academic Press;1998. p.92118.

14) Wielkiewicz RM. Interpreting low scores on the WISC-R third factor: it's more than distractibility. Psychol Assess 1990;2:91-97.

15) Anastopoulos AD, Spisto MA, Maher MC. The WISC-III freedom from distractibility factor: its utility in identifying children with attention deficit hyperactivity disorder. Psychol Assess 1994;6: 368-371.

16) Wechsler $D$. Wechsler Intelligence Scale for Children-fourth edition (WISC-IV). San Antonio, TX: Psychological Corporation;2003.

17) Bremner D, McTaggart B, Saklofske DH, Janzen T. WISC-IV GAI and CPI in psychoeducational assessment. Can J Sch Psychol 2011; 26:209-219.

18) Devena SE, Watkins MW. Diagnostic utility of WISC-IV general abilities index and cognitive proficiency index difference scores among children with ADHD. J Appl Sch Psychol 2012;28:133154.

19) Weiss LG, Gabel AD. WISC-IV technical report \#6: using the cognitive proficiency index in psychoeducational assessment [cited 2016 Aug 10]. Available from: https://www.pearsonclinical.com.au/files/ WISCIVTechReport6_CPI.pdf.

20) Kwak KJ, Oh SW, Kim CT. Korean-Wechsler Intelligence Scale for Children. 4th ed. Seoul: Hakjisa;2011.

21) Hur YA. Cognitive characteristics of potential high-risk ADHD children reflected on the K-WISC-IV. Ulsan: Univ. of Ulsan;2014.

22) Oh SH, Oh MY. Materials for beginners in K-WISC-IV workshop. Seoul: Hakjisa;2012.

23) Cohen J. A power primer. Psychol Bull 1992;112:155-159.

24) Weiss LG, Saklofske DH, Prifitera A, Holdnack JA. WISC-IV advanced clinical interpretation. New York: Academic Press;2006. p.142-156.

25) Prifitera A, Saklofske DH, Weiss LG. WISC-IV clinical use and interpretation. Amsterdam: Boston;2005. p.248-260.

26) Hwang ST, Kim JH, Park GB, Choi JY, Hong SH. Korean Wechsler Adult Intelligence Scale-4th edition (K-WAIS-IV). Daegu: Korea Psychology;2013. 